\section{Aurora kinase inhibitors: which role in the treatment of chronic myelogenous leukemia patients resistant to imatinib?}

\author{
Giovanni Martinelli,' \\ Cristina Papayannidis,' Ilaria lacobucci,' \\ Simona Soverini,' Daniela Cilloni, ${ }^{2}$ \\ Michele Baccarani' \\ Institute of Hematology and Medical \\ Oncology "L. and A. Seràgnoli", \\ University of Bologna, Italy \\ ${ }^{2}$ Division of Hematology and Internal \\ Medicine, Department of Clinical and \\ Biological Science, University of Turin, \\ Italy
}

\section{Abstract}

At present, there are no compounds in clinical development in the field of chronic myeloid leukemia (CML) or Philadelphia-positive $\left(\mathrm{Ph}^{+}\right)$acute lymphoblastic leukemia (ALL) that have been documented to harbor significant activity against the imatinib-resistant T315I mutation. Recent reports on the preclinical activity of some emerging tyrosine kinase inhibitors such as 0N012380, VX-680 and PHA-739358 promise possible clinical efficacy against this specific Bcr-Abl mutant form. Here, we focus on the role of aurora kinase inhibitor VX-680 and PHA-739358 in blocking the leukemogenic pathways driven by wildtype and T315I-Bcr-Abl in CML or $\mathrm{Ph}^{+}$ALL by reviewing recent research evidence. We also discuss the possibility of employing aurora kinase inhibitors as a promising new therapeutic approach in the treatment of CML and $\mathrm{Ph}^{+}$ALL patients resistant to first and second generation TK inhibitors.

\section{Introduction}

The molecular signature of chronic myeloid leukemia (CML) and Philadelphia-positive $\left(\mathrm{Ph}^{+}\right)$acute lymphoblastic leukemia (ALL) is the Bcr-Abl hybrid gene, originating from a reciprocal $\mathrm{t}(9 ; 22)$ chromosomal translocation on the $22 q$ - derivative, commonly referred to as the Philadelphia chromosome. ${ }^{1}$ The resulting fusion protein, Bcr-Abl, displays deregulated tyrosine kinase activity and drives CML. ${ }^{2}$ The disease begins with an indolent chronic phase (CP) marked by the expansion of myeloid cells with normal differentiation, and then inexorably proceeds to advanced phases, i.e., accelerated phase (AP) and the terminal blastic phase (BP). Imatinib (Gleevec, Glivec; STI571), a relatively selective tyrosine kinase inhibitor that blocks the catalytic activity of Bcr-Abl, is now the first-line treatment for all newly diagnosed CML patients. Despite excellent clinical results, there is still a need to improve therapy for patients with CML and $\mathrm{Ph}^{+}$ALL. More than $80 \%$ of newly diagnosed CML patients treated with imatinib in CP achieve a complete cytogenetic remission, as typified by the absence of the Philadelphia chromosome at the examination of 20 bone marrow meta-phases. ${ }^{3}$ However, residual Bcr$\mathrm{Abl}$ transcripts persist in the majority of these patients, as assessed by sensitive assays such as nested reverse transcription-polymerase chain reaction, and represent the potential pool from which disease recurrence may originate. While responses in CML in CP patients have been shown to last more than five years, ${ }^{3}$ most responding patients with $\mathrm{AP}$ - and $\mathrm{BP}$ $\mathrm{CML}$, as well as those with $\mathrm{Ph}^{+} \mathrm{ALL}$, relapse early despite continued therapy. Resistance to imatinib is most commonly mediated by $\mathrm{Abl}$ kinase domain mutations. ${ }^{4}$ We and other authors have reported that approximately half CML patients have evidence of point mutations within the Abl kinase domain at the time of resistance to imatinib. Mutations target critical contact points between imatinib and Bcr-Abl or, more often, induce a conformation to which imatinib is unable to bind. ${ }^{5}$ In the remaining patients, the reasons for imatinib resistance have to be traced to BcrAbl gene amplification or overexpression, clonal cytogenetic evolution, or altered levels of transport molecules responsible for imatinib influx and efflux ( $\mathrm{ABC}$ transporters, h0CT1). ${ }^{4}$

Abl mutations are at present the most extensively investigated and best characterized mechanism of resistance to imatinib. So far, at least 90 different point mutations have been isolated from relapsed CML patients who are resistant to imatinib. ${ }^{6-7}$ The clinical and pathogenetic impact of mutations varies according to their different degree of residual sensitivity to imatinib. Indeed, while certain Bcr-Abl mutations retain in vitro sensitivity to imatinib at physiologically relevant concentrations and therefore may not be clinically meaningful, others require increased doses of imatinib, and some confer a highly resistant phenotype (Table 1). ${ }^{9}$

\section{The T315I mutation is highly resistant to imatinib}

An amino acid substitution occurring at the so-called "gatekeeper" residue, i.e. threonine 315 , has attracted particular inter-
Correspondence: Giovanni Martinelli, Institute of Hematology and Medical Oncology "Seràgnoli", University of Bologna,

via Massarenti, 9 - 40138 Bologna, Italy

E-mail: giovanni.martinelli2@unibo.it

Key words: chronic myeloid leukemia, Bcr-Abl, imatinib, resistance, mutations, dasatinib, nilotinib, inhibitors.

Funding: this study was supported by European LeukemiaNet, COFIN 2003 (Molecular therapy of Ph-positive leukemias), FIRB 2001, A.I.L., A.I.R.C., Fondazione del Monte di Bologna e Ravenna, Italy.

Received for publication: 16 December 2008. Revision received: 13 January 2009.

Accepted for publication: 2 March 2009.

This work is licensed under a Creative Commons Attribution 3.0 License (by-nc 3.0).

(C) Copyright Giovanni Martinelli et al., 2009

Licensee PAGEPress, Italy

Hematology Reviews 2009; 1:e1

doi:10.4081/hr.2009.e1

est since it confers a high level of resistance not only to imatinib therapy but also to all of the newly developed tyrosine kinase inhibitors entered in clinical trials. Co-crystal structure analysis indicates that, on binding, the hydroxyl group of threonine 315 forms a crucial hydrogen bond with imatinib. ${ }^{10}$ Moreover, the side chain of threonine also sterically controls the binding of the inhibitor to hydrophobic regions adjacent to the ATPbinding site. ${ }^{11}$ In 10-15\% of imatinib-resistant patients, especially those in more advanced phases of disease, a threonine to isoleucine amino acid substitution may be observed. The T315I abrogates imatinib binding because it disrupts the above mentioned hydrogen bond and introduces a bulkier isoleucine side chain into the gatekeeper position. ${ }^{12}$ However, this explanation is not the most up-to-date. In fact, as recently demonstrated, the T315I resistance to imatinib mainly results from the breakdown of interactions between imatinib and both E286 and $\mathrm{M} 290 .{ }^{13}$ As a result, biochemical and cellular IC ${ }^{50}$ values of imatinib for the T315I-BcrAbl have been shown to be $>6400$ times higher than those of wild-type Bcr-Abl (Table 1). ${ }^{9}$ Some authors have suggested that the T315I is associated with highly aggressive disease phenotype and poor outcome if no timely therapeutic reassessment is made. ${ }^{14,15}$ However, the effects of the T315I mutation on kinase activity in vitro and transforming efficiency of Bcr-Abl in vitro and in vivo have been very recently investigated, suggesting that in the absence of imatinib, there is nei- 
ther increased kinase activity nor any growth advantage for cells carrying T315I-Bcr-Abl as compared to wild-type Bcr-Abl. ${ }^{5}$

\section{The two second-generation inhibitors in clinical development, dasatinib and nilotinib, are ineffective against the T315I mutant}

To counteract the problem of resistance due to point mutations, several second-generation inhibitors have been synthesized and tested in pre-clinical assays: nilotinib (AMN107), ${ }^{8,16-18}$ dasatinib (BMS-354825), ${ }^{8,19-23}$ bosutinib ${ }^{24}$ VX$680,{ }^{21,25}$ AP23464, ${ }^{26,27}$ bafetinib, ${ }^{28,29}$ PD166326, PD180970 and PD173955,,$^{10,30-32}$ and ON012380. ${ }^{33}$ Two of them are currently being evaluated in phase II clinical trials - the dualspecificity Src/Abl inhibitor dasatinib and the imatinib derivative nilotinib. Dasatinib is a novel, dual Src and Abl inhibitor entered in clinical trials. It has been shown to be $\sim 300$ times more potent than imatinib in Bcr-Abl inhibition assays. Excellent results in terms of hematologic and cytogenetic response in CML and $\mathrm{Ph}^{+}$ALL patients resistant to imatinib have been reported after dasatinib administration. ${ }^{34}$ Pre-clinical studies have demonstrated that dasatinib is active against at least fourteen imatinib-resistant Bcr-Abl mutants (M244V, G250E, Q252H/R, Y253F/H, E255K/N,
F317L, M351T, E355G, F359V, H396R, F486S). ${ }^{19}$ The only imatinib-resistant Bcr-Abl isoform that was clearly insensitive to dasatinib was the T315I mutant, which retained kinase activity even in the presence of micromolar concentrations of the compound (Table 1). ${ }^{19}$ Accordingly, imatinib-resistant patients harboring the T315I mutation have been shown not to benefit from dasatinib in the recent phase I trial. ${ }^{34}$

Nilotinib is a close relative of imatinib with more than 20-fold improved affinity for wildtype Bcr-Abl. ${ }^{16}$ It is highly efficacious in patients with imatinib-resistant $\mathrm{Ph}^{+} \mathrm{CML}$. In vitro experiment with cell lines transformed with mutated forms of Bcr-Abl showed IC ${ }^{50}$ proliferation inhibition for most mutations with the exception of the T315I, which remains refractory to nilotinib ${ }^{8}$ (Table 1). Accordingly, clinical responses have been observed in patients with various imatinib-resistant BcrAbl mutations but not in patients positive for the T315I in the recent phase I trial. ${ }^{35}$

Despite the pressing need for a clinically effective T315I-Bcr-Abl inhibitor, relatively few pre-clinical candidates have been reported. A potential pitfall might be the tendency to screen initially for Abl kinase inhibition rather than for T315I-specific inhibition. A promising approach is to design inhibitors targeting other regions of Bcr-Abl. For example, ON012380, a putative substrate-competitive inhibitor, exhibited low nanomolar activi- ty against imatinib-resistant Bcr-Abl mutants, including the T315I, in biochemical and cellular assays. ${ }^{33}$

\section{Aurora kinases as targets for cancer}

Between these new promising drugs, VX680 and PHA-739358, two aurora kinase A, B and $\mathrm{C}$ inhibitors, have a leading place. The aurora kinases are a family of serine/threonine kinases involved in many cellular functions, including progression through mitosis, by regulating spindle formation, chromosome segregation and cytokinesis. ${ }^{35-37}$ The overexpression of aurora kinases has been reported in many human solid tumors, leading to defects in centrosome function, aberrant spindle assembly, misalignment of chromosomes, abnormal cytokinesis and genetic instability, determining the activation of oncogenic pathways. ${ }^{38-40}$ Many authors reported an aberrant expression of the aurora A and B kinases also in leukemia cells, suggesting a potential role of these molecular targets in the treatment of CML and ALL. ${ }^{4142}$ Aurora kinase function is mediated by the phosphorylation of several substrates that have important roles in cell division, such as proteins survivin, CENP-A and serine 10 on histone $\mathrm{H} 3{ }^{37}$ The aurora kinases range in size from 309 to 403 amino acids. They have a $\mathrm{C}$ terminal domain that is

Table 1. Comparison between imatinib, dasatinib and nilotinib IC50 values obtained in Ba/F3 cellular proliferation assays. Adapted from [8].

\begin{tabular}{|c|c|c|c|c|c|c|}
\hline \multirow{2}{*}{ Abl variant } & \multicolumn{5}{|c|}{ Cellular proliferation } & \multirow{2}{*}{$\begin{array}{l}\text { Dasatinib } \\
\text { fold-change }\end{array}$} \\
\hline & $\mathrm{IC}_{50}(\mathrm{nM})$ & Fold-change & $\mathrm{IC}_{50}(\mathrm{nM})$ & Fold-change & $\mathrm{IC}_{50}(\mathrm{nM})$ & \\
\hline Wild-type & 260 & 1 & 13 & 1 & 0.8 & 1 \\
\hline M244V & 2,000 & 8 & 38 & 3 & 1.3 & 2 \\
\hline G250E & 1,350 & 5 & 48 & 4 & 1.8 & 2 \\
\hline $\mathrm{Q} 252 \mathrm{H}$ & 1,325 & 5 & 70 & 5 & 3.4 & 4 \\
\hline Y253F & 3,475 & 13 & 125 & 10 & 1.4 & 2 \\
\hline $\mathrm{Y} 253 \mathrm{H}$ & $>6,400$ & $>25$ & 450 & 35 & 1.3 & 2 \\
\hline $\mathrm{E} 255 \mathrm{~K}$ & 5,200 & 20 & 200 & 15 & 5.6 & 7 \\
\hline $\mathrm{E} 255 \mathrm{~V}$ & $>6,400$ & $>25$ & 430 & 33 & 11 & 14 \\
\hline F311L & 480 & 2 & 23 & 2 & 1.3 & 2 \\
\hline T315I & $>6,400$ & $>25$ & $>2000$ & $>154$ & $>200$ & $>250$ \\
\hline F317L & 1,050 & 4 & 50 & 4 & 7.4 & 9 \\
\hline M351T & 880 & 3 & 15 & 1.2 & 1.1 & 1.4 \\
\hline F359V & 1,825 & 7 & 175 & 13 & 2.2 & 3 \\
\hline L387M & 1,000 & 4 & 49 & 4 & 2 & 3 \\
\hline H396P & 850 & 3 & 41 & 3 & 0.6 & 0.8 \\
\hline H396R & 1,750 & 7 & 41 & 3 & 1.3 & 2 \\
\hline
\end{tabular}


responsible for regulation of the protein levels via proteasomal degradation; a highly conserved catalytic domain; and a short N-terminal domain that varies in length between the kinases and contributes to the differing locations of the kinases within cells. ${ }^{43}$ (Figure 1).

The aurora A isotype (also known as aurora, Aurora-2, AIK, AIR-1, AIRK1, AYK1, BTAK, Eg2, MmIAK1 and STK15) is widely expressed in proliferating normal tissues, with expression being cell-cycle-dependent and peaking at the G2/M point of the cell cycle. During mitosis, the kinase is virtually confined to the spindle poles, where it is needed for centrosome separation and maturation. ${ }^{44}$ An overexpression of aurora A causes an increase in centrosome numbers and aneuploidy, ${ }^{45}$ leading to the transformation of mammalian cells and also causes resistance to apoptosis induced by taxol in human cancer cell lines. Moreover, this kinase is a key regulatory component of the p53 pathway as its overexpression leads to increased p53 degradation, which facilitates oncogenic transformation. ${ }^{46}$ Human aurora A has been proposed as a "drugable" target in several tumors including pancreatic, ${ }^{46,47}$ hepatocellular, ${ }^{48}$ breast, ${ }^{49}$ nonendometriod, ${ }^{50}$ and ovarian carcinomas ${ }^{51}$ gliomas ${ }^{52}$ and aggressive nonHodgkin's lymphoma. ${ }^{53}$ Aurora B (also known as Aurora-1, AIM-1, AIK2, AIR-2, AIRK-2, ARK2, IAL-1 and STK12) activity is required for bipolar chromosome orientation and condensation. ${ }^{54}$ Aurora B kinases are chromosomal passenger proteins, which are found in cells in a complex with inner centromere protein (INCENP) and survivin. The overexpression of an aurora B kinase-dead mutant (K-R) causes multiple defects in the mitotic machinery, including the loss of kinetochore attachment to microtubules and the exit from mitosis without anaphase or cytokinesis. ${ }^{55}$ Increased Aurora B expression correlates with increased grade in glioma and colon cancer ${ }^{56-57}$ and with anaplasia in thyroid carcinoma. ${ }^{58}$ Aurora C (also known as AIK3) expression plays a role in spermatogenesis at the time when cells assemble the two meiotic spindles and also cooperates with aurora B to regulate mitotic chromosome dynamics in mammalian cells..$^{59}$ Aurora $\mathrm{C}$ overexpression has been detected in tumor cell lines in vitro ${ }^{60}$ and in biopsy samples from colorectal carcinoma. ${ }^{61}$

\section{Novel aurora kinase inhibitors effective against the T315I- Bcr-Abl}

Several compounds have been pre-clinically screened for their inhibitory activity against aurora kinases (VX680, MLN8054, AZD1152, R766, R763, PHA-739358, AT9283) and many of

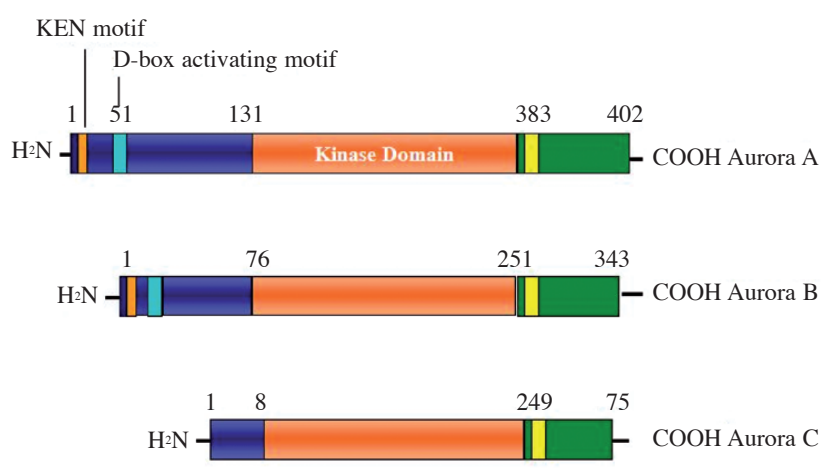

Figure 1. Schematic representation of domain organization of aurora kinases. Aurora kinases have three domains: the $\mathrm{N}$-terminal and $\mathrm{C}$-terminal domains which contain most of the aurora's regulatory motifs and the catalytic domain in the central region. The alignment of auroras A and B allows the identification of one distantly conserved 'KEN' motif, spanning 11-18 residues. The 'KEN' motif acts as a $\mathrm{Cdh}^{-}$dependant anaphase-promoting complex recognition signal.

Table 2. Novel compound aurora-kinase inhibitors in clinical trial development.

\begin{tabular}{llll}
\hline TK inhilbitor & Company & Phase & Target(s) \\
MK-0457 & Merck & I-II & Aurora, FLT-3, JAK-2 \\
PHA-739358 & Nerviano & II & Aurora \\
\hline KW-2449 & Kyowa & I & Aurora \\
Deciphera & Decifera & I & Abl \\
\hline AS703569 & Merck Serono & I-II & Aurora, Abl, JAK-2 \\
AZD1152 & Astra-Zeneca & I-II & Aurora \\
\hline
\end{tabular}

Table 3. Comparison between the binding affinity of imatinib and of the aurora kinase inhibitors BIRB-796 and MK-0457 for wild-type and drug-resistant Abl variants. Adapted from [21].

\begin{tabular}{lrrr} 
Abl variant & Imatinib & BIRB-796 & MK-0457 \\
Wild-type & 2 & 2,000 & 20 \\
Q252H & 20 & 4,000 & 10 \\
\hline Y253F & 40 & 2,000 & 20 \\
E255K & 100 & $>10,000$ & 50 \\
\hline M351T & 10 & 2,000 & 8 \\
F359V & 20 & 8,000 & 20 \\
\hline T315I & 6,000 & 40 & 5 \\
H396P & 60 & $>10,000$ & 7 \\
\hline
\end{tabular}

Table 4. Comparison between the binding affinity of imatinib and of the aurora kinase inhibitors PHA-739358 and MK-0457 for wild-type and drug-resistant Abl variants. Adapted from [63].

\begin{tabular}{lccrc} 
& WI & $\begin{array}{r}\text { E255V } \\
(\text { P loop })\end{array}$ & T315I & M351T \\
Imatinib & 0.230 & 0.610 & $>20.000$ & 0.100 \\
PHA-739358 & 0.021 & 0.014 & 0.005 & 0.015 \\
\hline MK-0457 & 0.083 & 0.205 & 0.085 & 0.045 \\
\hline
\end{tabular}


them are being tested in clinical phase I/I trials (Table 2). MK-0457 (VX-680) is a pan-aurora kinase inhibitor with demonstrated in vitro activity against wild-type and mutated Bcr-Abl, including the T315I form, as well as FLT3 and JAK-2. ${ }^{21}$ Fascinatingly, Carter et al. have found that the aurora kinase inhibitor VX-680, already in phase I trials, and the p38 inhibitor BIRB-796, in clinical trials for inflammatory disease, inhibit the imatinib- and dasatinibresistant T315I-Bcr-Abl with high affinity (Tables 3 and 4). In fact, contrasting results related to this compound have been published. In particular, BIRB-796 binds with good affinity to T315I-Bcr-Abl ( $\mathrm{Kd}=40 \mathrm{nM})$, but has significantly weaker affinity for wild-type and other imatinib-resistant forms of Abl, with Kd values $>1 \mu \mathrm{M}^{21}$ In contrast, as reported by other authors, the compound fails to inhibit the proliferation of cells expressing T315I, suggesting a lack of clinical benefit for patients harboring such a mutation. ${ }^{22}$

In a recent phase I-II study, MK-0457 was shown to be active in patients with T315I phenotype-refractory CML or Ph-positive ALL, with no significant extramedullary toxicity. ${ }^{62}$ Because of a potential heart safety issue revealed in one patient who experienced QTc prolongation, the enrolment on phase II protocol was halted in November 2007. Furthermore, an innovative phase I clinical study of sequential and concomitant treatment with dasatinib and MK-0457 has been conducted, based on the suggestion that such a combinatory approach would suppress the emergence of T315I and other resistant clones, improving upon the response rate for dasatinib and the durability of response. To date, 3 patients with wild-type chronic myeloid leukemia (CML) or Ph-positive acute lymphoblastic leukemia (ALL) have been enrolled, and this innovative therapeutic combination showed a relevant hematologic activity and a good safety profile. PHA-739358 is a small molecule that selectively inhibits the ATP site of Aurora-A ( $\mathrm{IC}^{50}=13$ $\mathrm{nM})$ and Aurora-B ( $\left.\mathrm{IC}_{50}=79 \mathrm{nM}\right)$ kinases. $^{63}$ Starting from the rationale that aurora kinases play an important role in mitosis and that the interruption of their function has significant potential in the treatment of cancer, the drug, formulated for intravenous infusion, is being developed for therapeutic use in solid tumors and in patients with Philadelphia positive leukemias. Interestingly, PHA-739358, when tested against a panel of more than 30 kinases, has shown a strong cross-reactivity with c$\mathrm{Abl}\left(\mathrm{IC}^{50}=25 \mathrm{nM}\right)$. Its inhibitory activity on $\mathrm{ABL}$ in cells was confirmed in K562 leukemia cells which bear the Philadelphia chromosome related translocation Bcr-Abl. Furthermore PHA-739358 inhibits phosphorylation of
Tyr412, which is located in the kinase activation loop of Abl and is also active against the T315I mutant of Abl, which is resistant to other ATP competitive inhibitors in the clinic, such as gleevec, and second generation TK inhibitors. A multicentric phase I/II study, aimed to test PHA-739358 in patients with chronic, accelerated or blast phase CML relapsing on gleevec or c-Abl therapy and preferably with T315I mutation in Bcr-Abl kinase is ongoing.

\section{Binding mode of VX-680 and PHA-739358 to Abl}

The compound VX-680, developed by Vertex Pharmaceuticals as an inhibitor of the aurora kinases, is a Y-shaped molecule, with a $\mathrm{N}$-methyl-piperazine group forming the base or leg of the "Y", a pyrimidine group at the fork, and a methylpyrazole group at one arm and a substituted phenyl group at the other

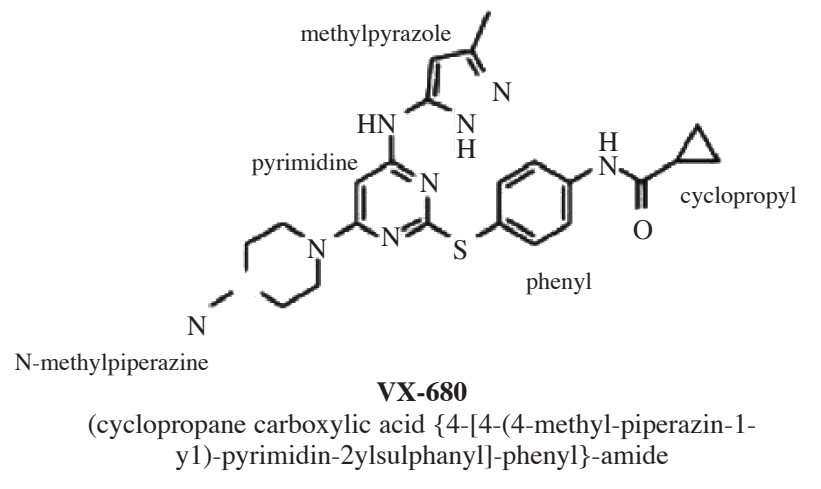

Figure 2. Chemical structure of VX-680 aurora kinase inhibitor. [Reprinted and adapted with permission from http://kinasepro.wordpress.com].

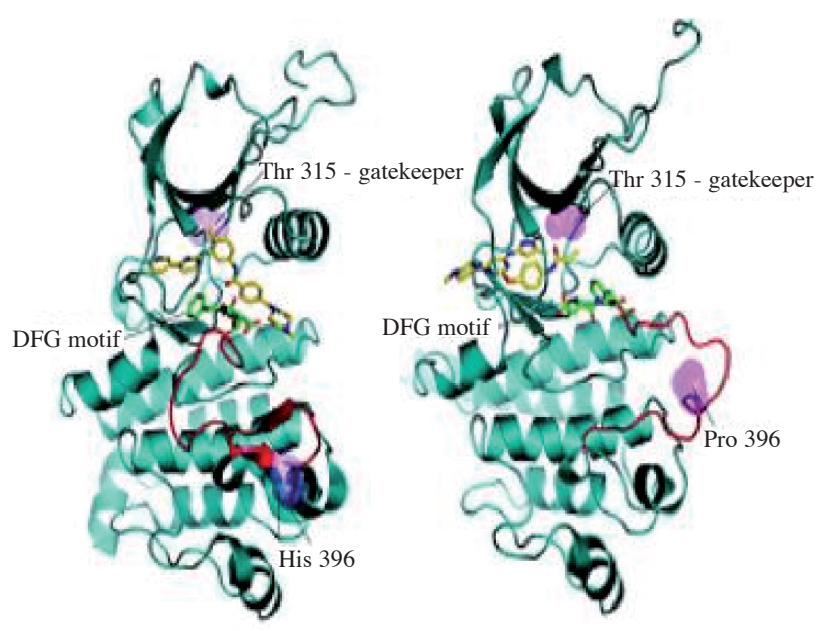

Abl: Imatinib

Abl: VX-680

Figure 3. Structure of Abl domain kinase bound to imatinib (left) and to VX-680 (right). [Reprinted and adapted with permission of AACR from: Young MA, et al. Structure of the kinase domain of an imatinib-resistant Abl mutant in complex with the Aurora kinase inhibitor VX-680. Cancer Res 2006 Jan 15;66(2):1007-14]. 
arm (Figure 2). A recent study ${ }^{25}$ showed that VX-680 forms a hydrogen bond with the strictly conserved Asp381 of the Asp-Phe-Gly (DFG) motif in the Abl kinase domain and maintains it in an orientation close to one that is normally seen in active kinases, although the activation loop of $\mathrm{Abl}$ is not phosphorylated in this structure (Figure 3). Furthermore, VX-680 does not deeply penetrate into the kinase domain as imatinib does and it is anchored to it by four hydrogen bonds. Three of these are formed between two carbonyl groups (Glu316 and Met318) and an amide nitrogen (Met318) in the "hinge region" of the kinase and three nitrogen atoms, one in the linker between the pyrimidine group and the methylpyrazole group, and the other two in the methylpyrazole group. These bonds are a common feature of kinase inhibitors and are independent of the sequence of the kinase..$^{59}$ Likewise, the fourth hydrogen bond, made by VX-680 to the side chain of Asp381 of the DFG motif, is to a strictly invariant catalytic residue. Using these four anchors, the inhibitor makes contact with 14 side chains within the kinase domain, eight of which are identical between Abl and aurora. One of the non-conservative substitutions is at the gatekeeper position, where Thr315 in Bcr-Abl is replaced by Leu210 in aurora A kinase (Figure 4). The side chains of isoleucine (at position 315 of Bcr-Abl) and leucine (at position 210 of aurora A kinase) can be accommodated readily between the two sides of the "Y" of VX-680. For this reason, VX680 , in contrast to imatinib, is able to inhibit the kinase activity of both wild-type Bcr-Abl and T315I-Bcr-Abl. To understand the structural basis of the capability of PHA-739358 to bind and inhibit the T315I mutant, the crystal structure of the inhibitor-protein complex was determined $^{63}$ (Figure 5). The protein is in the typical conformation of active kinases, with the activation loop in the extended DFG "in" conformation. Indeed, Asp381 points into the active site and interacts with $\mathbf{M g}^{2+}$ ion that occupies a position similar to the one usually seen in the structures of kinases in complex with ATP. The glycine loop adopts an extended conformation, in contrast to the other publicly available Abl structures where the loop is more distorted, which could be due to the specific binding mode of our inhibitor. The purified T315I Abl kinase domain used for crystallization experiments is predominantly phosphorylated on the activation loop at Tyr393, whereas Tyr253, Tyr257, and Tyr264 are phosphorylated at lower levels. These interactions probably stabilize the active conformation of the activation loop, which is, however, very similar to the structures reported for dasatinib in complex with the WT Abl kinase domain ${ }^{64}$ and of MK-0457 in complex with the Abl mutant H396P. ${ }^{25}$ The mutation of the threonine to the more bulky isoleucine does not seem to cause any widespread conformational changes but creates a steric hindrance that would interfere with the binding of inhibitors, such as imatinib, nilotinib, and dasatinib, which make use of the hydrophobic pocket. The binding mode of PHA-739358 is very similar to that reported for the complex of the same compound with aurora A (Figure 5B and
D), although the conformation of the proteins around the ATP-binding site shows some differences because in the aurora A structure the DFG motif is more similar to the "out" conformation. However, all of the essential contacts between PHA-739358 and Abl T315I involve highly conserved elements. The molecule makes three hydrogen bonds with the protein

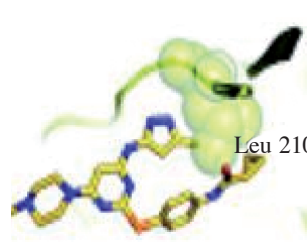

Aurora: VX-680 (model)

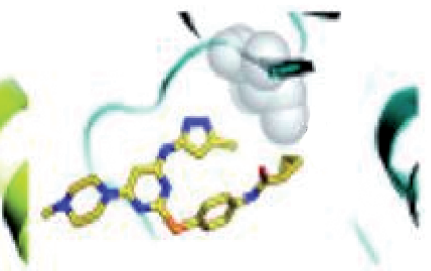

$+$

T315I-Bcr-Abl: VX-680 (model)

Figure 4. Binding mode of VX-680 to aurora A and T315I-Bcr-Abl. Both leucine $\mathrm{e}^{210}$ and isoleucine $e^{315}$ side chains can be accommodated between the two arms of the "Y" of VX680. [Reprinted and adapted with permission of AACR from: Young MA, et al. Structure of the kinase domain of an imatinib-resistant Abl mutant in complex with the Aurora kinase inhibitor VX-680. Cancer Res 2006 Jan 15;66(2):1007-14].

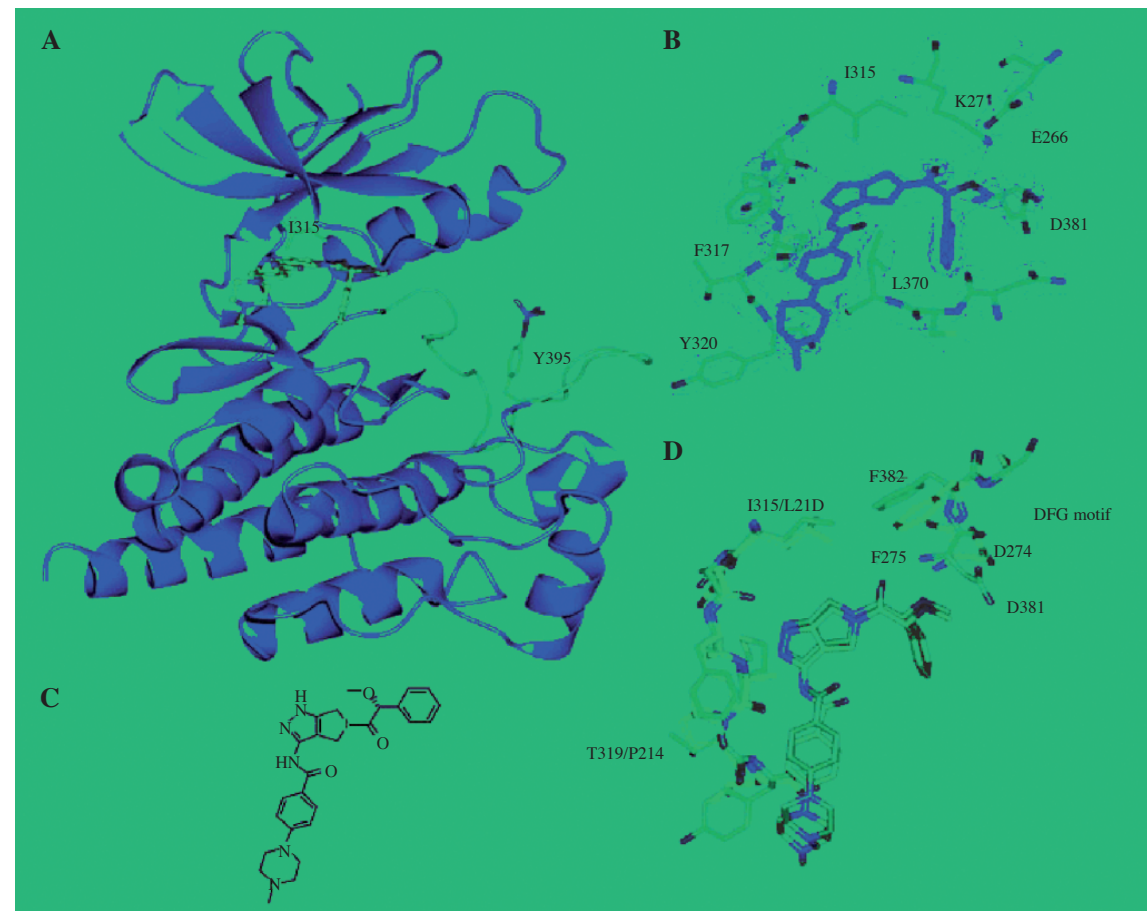

Figure 5. Structure of Abl-T315I-PHA 739358 complex. (A) Ribbon representation of the structure of T315I Abl mutant with PHA-739358. The mutated gatekeeper residue Ile315 and the activation loop with the phosphorylated residue Tyr393 are highlighted in green. (B) Close-up view of the binding site of PHA-739358 showing the final 2 FoFc electron density map, contoured at $1 \mathbf{j}$, associated with the ligand. (C) Chemical formula of PHA 739358. (D) Comparison of PHA-739358 complexes with the aurora A structure. Details of the binding of PHA-739358 to Abl (green carbon atoms) and to aurora $\mathrm{A}$ (yellow carbon atoms) showing the residues of the hinge region and of the DFG motif of both proteins. [Reprinted and adapted with permission of AACR from: Modugno M, et al. Crystal structure of the T315I Abl mutant in complex with the aurora kinases inhibitor PHA-739358. Cancer Res 2007 Sep 1;67(17):7987-90]. 


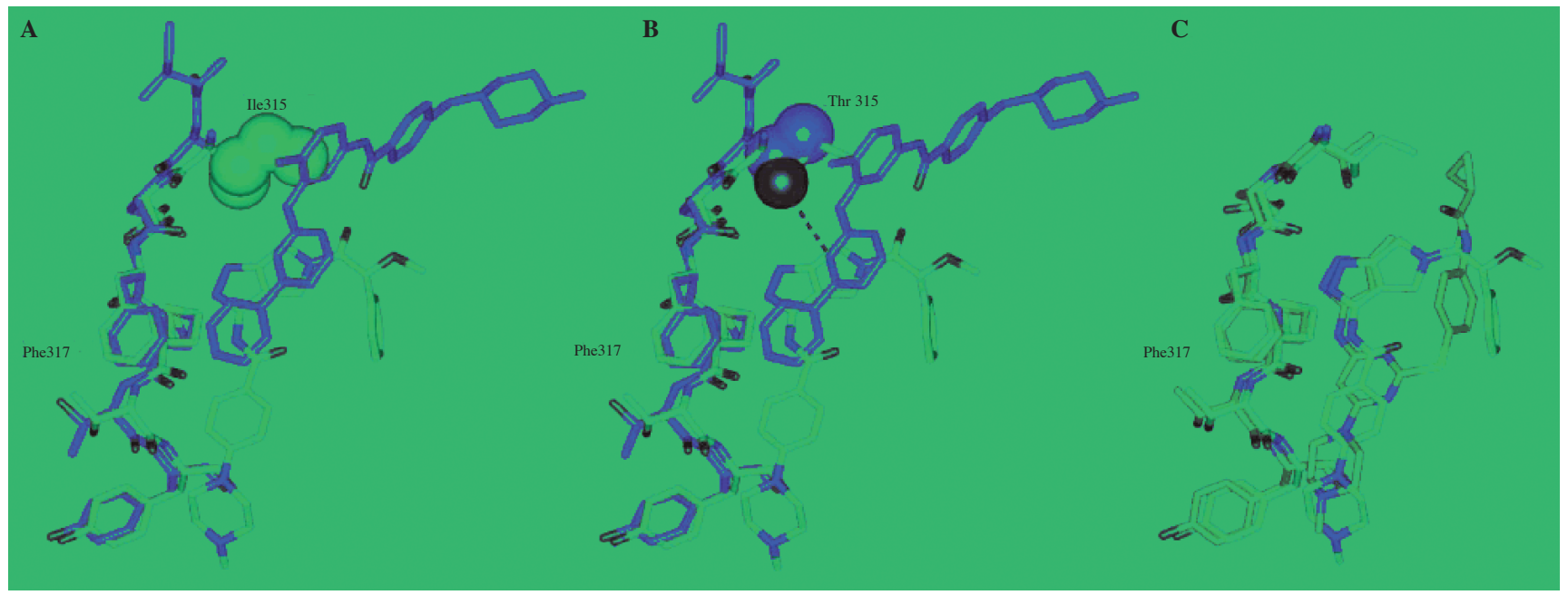

Figure 6. Comparison of PHA-739358 complex with imatinib and MK-0457 complexes. (A) and (B) PHA-739358 complex (green carbon atoms) superimposed with the structure of imatinib (magenta carbon atoms). The gatekeeper residues Ile315 of PHA-739358 complex (A) and Thr315 of the imatinib complex (B) are shown with van der Waals spheres. In the T315I mutant, the isoleucine side chain causes a steric clash with imatinib; in addition, the hydrogen bond between imatinib and the side chain oxygen of threonine is lost. (C) Structure of Abl-PHA-739358 complex (green carbon atoms) superimposed on the structure of MK-0457. [Reprinted and adapted with permission of AACR from: Modugno M, et al. Crystal structure of the T315I Abl mutant in complex with the aurora kinases inhibitor PHA-739358. Cancer Res 2007 Sep 1;67(17):7987-90].

backbone of the hinge region: the two nitrogen atoms of the pyrrolopyrazole core interact with the carbonyl oxygen of Glu316 and with the amide nitrogen of Met318, whereas the nitrogen of the amide group hydrogen bonds to the carbonyl oxygen of Met318. In addition, the side chain nitrogen of the conserved Lys271 is within hydrogen bonding distance of the oxygen of the carbonyl group and the oxygen of the methoxy group. As in the aurora structure, the benzyl group packs against Leu370 (Leu263 in aurora), whereas the $\mathrm{N}$ methyl-piperazine points toward the solvent accessible area of the kinase pocket. The gatekeeper residue in the aurora kinases is Leu210, a large and hydrophobic residue very similar to isoleucine, and we have observed that PHA-739358 binds in the ATP-binding pocket of aurora A without any steric hindrance with the gatekeeper residue. Indeed, the co-crystal structure reported here reveals that the compound is bound to the Abl T315I kinase domain in a way that accommodates the substitution of isoleucine for threonine. Figure 6 shows the structure of the Abl T315I complex with PHA-739358 superimposed on those of the Abl WT with imatinib and Abl H396P with MK-0457. In the T315I mutant, the isoleucine side chain causes a steric clash with imatinib and the hydrogen bond between imatinib and the side chain oxygen of threonine is lost (Figure 6A and B). On the contrary, both PHA-739358 and MK-0457 bind in such a way to avoid the gatekeeper residue (Figure 6C) and this provides an explanation for the ability of both compounds to accommo- date the isoleucine substitution. Furthermore, the pyrrolopyrazole scaffold of PHA739358 is situated within van der Waals distance of the side chain of Ile315 mimicking the interaction between the inhibitor and Leu210 in aurora A. It is possible that this favorable hydrophobic packing interaction may explain why PHA-739358 is more active against the mutant than the WT protein. PHA739358 could represent a valuable novel agent to target the T315I Bcr-Abl mutation, and preclinical and clinical data are coming through to support this concept.

\section{Conclusions}

The T315I is responsible for approximately $15 \%$ of the cases of relapse in CML and $\mathrm{Ph}^{+}$ ALL patients on imatinib therapy. The clinical relevance of this mutant is likely to increase considerably as to date it seems to represent the main mechanism of resistance to dasatinib and nilotinib, the second-generation inhibitors already being developed clinically. Structural analyses indicate that the substitution of threonine with isoleucine at residue 315 eliminates a crucial hydrogen-bonding interaction and introduces a steric clash which abrogates binding and effective inhibition of Bcr-Abl by imatinib as well as by several novel inhibitors. A possible approach to the development of second-line strategies overcoming resistance induced by the T315I mutation is to design inhibitors binding regions of
Bcr-Abl other than the ATP binding pocket. An intriguing alternative is to explore the possibility of whether molecules that have been developed as inhibitors for other protein kinases and are already undergoing clinical trials might include the T315I-Bcr-Abl mutant among their off-targets. Although off-target activity may lead to undesirable side effects, it has to be recognized that focusing on compounds that are already being tested in clinical practice may speed up the development of successful therapeutic strategies. Recent studies have shown that MK-0457 (VX-680) and PHA739358, two small-molecule aurora kinase inhibitors, have in vitro activity against the T315I-Bcr-Abl. Moreover, preliminary data showed promising clinical efficacy in patients affected by Philadelphia positive leukemias, relapsing or resistant to first and second generation TK inhibitors. Such a remarkable efficacy raises the question of whether aurora kinases may also harbor some pathogenetic significance in CML and/or $\mathrm{Ph}^{+}$ALL or may be selectively deregulated by the T315I-Bcr-Abl, and whether auroras may be a suitable secondary target for inhibition.

\section{References}

1. Bartram CR, de Klein A, Hagemeijer A, van Agthoven T, Geurts van Kessel A, Bootsma D, et al. Translocation of c-abl oncogene correlates with the presence of a Philadelphia chromosome in chronic myelocytic leukaemia. Nature 
1983;306:277-80.

2. Groffen J, Stephenson JR, Heisterkamp N, Bartram C, de Klein A, Grosveld G. The human c-abl oncogene in the Philadelphia translocation. J Cell Physiol Suppl 1984; 3:179-91.

3. Druker B, Guilhot F, O'Brien SG, Larson R. Long-term benefits of imatinib (IM) for patients newly diagnosed with chronic myelogenous leukemia in chronic phase (CML-CP): the 5-year update from the IRIS study. J Clin Oncol 2006 ASCO Annual Meeting Proceedings 2006;24:6506.

4. Hochhaus A, Hughes T. Clinical resistance to imatinib: mechanisms and implications. Hematol Oncol Clin North Am 2004; 18:641-56, ix.

5. Soverini S, Colarossi S, Gnani A, et al. Frequency, distribution and prognostic value of ABL kinase domain (KD) mutations in different subsets of Philadelphiapositive $(\mathrm{Ph}+)$ patients (pts) resistant to Imatinib (IM) by the GIMEMA Working Party on CML. Blood 2005;106:435.

6. Melo JV, Chuah C. Resistance to imatinib mesylate in chronic myeloid leukaemia. Cancer Lett 2007;249:121-32.

7. Branford S. Chronic myeloid leukemia: molecular monitoring in clinical practice. Hematology Am Soc Hematol Educ Program 2007;2007:376-83.

8. Golemovic M, Verstovsek S, Giles F, Cortes J, Manshouri T, Manley PW, et al. AMN107, a novel aminopyrimidine inhibitor of Bcr$\mathrm{Abl}$, has in vitro activity against imatinibresistant chronic myeloid leukemia. Clin Cancer Res 2005;11:4941-7.

9. O' Hare T, Walters DK, Stoffregen EP, Jia T, Manley PW, Mestan J, et al. In vitro activity of Bcr-Abl inhibitors AMN107 and BMS354825 against clinically relevant imatinib-resistant Abl kinase domain mutants. Cancer Res 2005;65:4500-5.

10. Corbin AS, La Rosee P, Stoffregen EP, Druker BJ, Deininger MW. Several Bcr-Abl kinase domain mutants associated with imatinib mesylate resistance remain sensitive to imatinib. Blood 2003;101:4611-4.

11. Nagar B, Bornmann WG, Pellicena P, Schindler T, Veach DR, Miller WT, et al. Crystal structures of the kinase domain of c-Abl in complex with the small molecule inhibitors PD173955 and imatinib (STI571). Cancer Res 2002;62:4236-43.

12. Gorre ME, Mohammed M, Ellwood K, Hsu $\mathrm{N}$, Paquette R, Rao PN, et al. Clinical resistance to STI-571 cancer therapy caused by BCR-ABL gene mutation or amplification. Science 2001;293:876-80.

13. Lee TS, Potts SJ, Kantarjian H, et al. Molecular basis explanation for imatinib resistance of BCR-ABL due to T315I and Ploop mutations from molecular dynamics simulations. Cancer 2008;112:1744-53.

14. Pricl S, Fermeglia M, Ferrone M, Tamborini E. T315I-mutated Bcr-Abl in chronic myeloid leukemia and imatinib: insights from a computational study. Mol Cancer Ther 2005; 4:1167-74.

15. Nicolini FE, Corm S, Le QH, Sorel N, Hayette S, Bories D, et al. Mutation status and clinical outcome of 89 imatinib mesylate-resistant chronic myelogenous leukemia patients: a retrospective analysis from the French intergroup of CML (Fi(phi)-LMC GROUP). Leukemia 2006; 20:1061-6.

16. Miething C, Feihl S, Mugler C, Grundler R, von Bubnoff N, Lordick F, et al. The Bcr-Abl mutations T315I and Y253H do not confer a growth advantage in the absence of imatinib. Leukemia 2006;20:650-7.

17. Weisberg E, et al. Characterization of AMN107, a selective inhibitor of native and mutant Bcr-Abl. Cancer Cell 2005;7: 129-41.

18. Weisberg E, Manley PW, Breitenstein W, Bruggen J, Cowan-Jacob SW, Ray A, et al. AMN107 (nilotinib): a novel and selective inhibitor of BCR-ABL. Br J Cancer 2006;94: 1765-9.

19. Shah NP, Tran C, Lee FY, Chen P, Norris D, Sawyers CL. Overriding imatinib resistance with a novel ABL kinase inhibitor. Science 2004;305:399-401.

20. Burgess MR, Skaggs BJ, Shah NP, Lee FY, Sawyers CL. Comparative analysis of two clinically active BCR-ABL kinase inhibitors reveals the role of conformationspecific binding in resistance. Proc Natl Acad Sci USA, 2005;102:3395-400.

21. Carter TA, Wodicka LM, Shah NP, Velasco AM, Fabian MA, Treiber DK, et al. Inhibition of drug-resistant mutants of ABL, KIT, and EGF receptor kinases. Proc Natl Acad Sci USA 2005;102:11011-6.

22. 0'Hare T, Druker BJ. BIRB-796 is not an effective ABL(T315I) inhibitor. Nat Biotechnol 2005;23:1209-10.

23. Tokarski JS, Newitt JA, Chang CY, Cheng JD, Wittekind M, Kiefer SE, et al. The structure of Dasatinib (BMS-354825) bound to activated ABL kinase domain elucidates its inhibitory activity against imatinib-resistant ABL mutants. Cancer Res 2006;66:5790-7.

24. Golas JM, Arndt K, Etienne C, Lucas J, Nardin D, Gibbons J, et al. SKI-606, a 4anilino-3-quinolinecarbonitrile dual inhibitor of Src and Abl kinases, is a potent antiproliferative agent against chronic myelogenous leukemia cells in culture and causes regression of K562 xenografts in nude mice. Cancer Res 2003;63:375-81.

25. Young MA, Shah NP, Chao LH, Seeliger M, Milanov ZV, Biggs WH 3rd, et al. Structure of the kinase domain of an imatinib-resist- ant Abl mutant in complex with the Aurora kinase inhibitor VX-680. Cancer Res 2006;66:1007-14.

26. O'Hare T,s Pollock R, Stoffregen EP, Keats JA, Abdullah OM, Moseson EM, et al. Inhibition of wild-type and mutant Bcr-Abl by AP23464, a potent ATP-based oncogenic protein kinase inhibitor: implications for CML. Blood 2004; 104:2532-9.

27. Azam M, Nardi V, Shakespeare WC, Metcalf CA 3rd, Bohacek RS, Wang Y, et al. Activity of dual SRC-ABL inhibitors highlights the role of BCR/ABL kinase dynamics in drug resistance. Proc Natl Acad Sci USA 2006;103:9244-9.

28. Kimura S, Naito H, Segawa H, Kuroda J, Yuasa T, Sato K, et al. New tyrosine kinase inhibitors in the treatment of chronic myeloid leukemia. Curr Pharm Biotechnol 2006;7:371-9.

29. Naito H, Kimura S, Nakaya Y, Naruoka H, Kimura S, Ito $S$, et al. In vivo antiproliferative effect of NS-187, a dual Bcr-Ab/Lyn tyrosine kinase inhibitor, on leukemic cells harbouring Abl kinase domain mutations. Leuk Res 2006; 30:1443-6.

30. Dorsey JF, Jove R, Kraker AJ, Wu J. The pyrido[2,3-d] pyrimidine derivative PD180970 inhibits p210Bcr-Abl tyrosine kinase and induces apoptosis of K562 leukemic cells. Cancer Res 2000;60:312731.

31. Huron DR, Gorre ME, Kraker AJ, Sawyers CL, Rosen N, Moasser MM, et al. A novel pyridopyrimidine inhibitor of abl kinase is a picomolar inhibitor of Bcr-abl-driven K562 cells and is effective against STI571resistant Bcr-abl mutants. Clin Cancer Res 2003;9:1267-73.

32. La Rosee P, Corbin AS, Stoffregen EP, Deininger MW, Druker BJ. Activity of the Bcr-Abl kinase inhibitor PD180970 against clinically relevant Bcr-Abl isoforms that cause resistance to imatinib mesylate (Gleevec, STI571). Cancer Res 2002;62: 7149-53.

33. Gumireddy K, Baker SJ, Cosenza SC, John P, Kang AD, Robell KA, et al. A non-ATPcompetitive inhibitor of BCR-ABL overrides imatinib resistance. Proc Natl Acad Sci USA 2005;102:1992-7.

34. Talpaz M, Shah NP, Kantarjian H, Donato $\mathrm{N}$, Nicoll J, Paquette R, et al. Dasatinib in imatinib-resistant Philadelphia chromosome-positive leukemias. N Engl J Med 2006; 354: 2531-41.

35. Keen N, Taylor S. Aurora-kinase inhibitors as anticancer agents. Nat Rev Cancer 2004;4: 927-36.

36. Marumoto T, Zhang D, Saya H. Aurora-A, a guardian of poles. Nat Rev Cancer 2005;5:42-50.

37. Carvajal RD, Tse A, Schwartz GK. Aurora kinases: new targets for cancer therapy. 
Clin Cancer Res 2006;12:6869-75.

38. Matthews N, Visintin C, Hartzoulakis B, Jarvis A, Selwood DL. Aurora A and B kinases as targets for cancer: will they be selective for tumors? Exppert Rev Anticancer Ther 2006;6:109-20.

39. Wang X, Zhou YX, Qiao W, et al. Overexpression of Aurora kinase A in mouse mammary epithelium induces genetic instability preceding mammary tumor formation. Oncogene 2006;25:7148-58.

40. Zhou H, Kuang J, Zhong L et al. Tumour ampilified kinase STK15/BTAK induces centrosome ampilification, aneuploidy and transformation. Nat Genet 1998;20:189-93.

41. Yang J, Ikezoe T, Nishioka C, Tasaka T, Taniguchi A, Kuwayama Y, et al. AZD1152, a novel and selective aurora B kinase inhibitor, induces growth arrest, apoptosis, and sensitization for tubulin depolymerizing agent or topoisomerase II inhibitor in human acute leukemia cells in vitro and in vivo. Blood 2007;110:2034-40.

42. Ikezoe T, Yang J, Nishioka C, Tasaka T, Taniguchi A, Kuwayama Y, et al. A novel treatment strategy targeting Aurora kinases in acute myelogenous leukemia. Mol Canc Ther 2007;6:1851-7.

43. Hu HM, Chuang CK, Lee MJ, Tseng TC, Tang TK. Genomic organization, expression, and chromosome localization of a third Aurora-related kinase gene, Aie1. DNA Cell Biol 2000;19:679-88.

44. Kufer TA, Nigg EA, Sillje HN. Regulation of Aurora-A kinase on the mitotic spindle. Chromo-soma 2003;112:159-63.

45. Meraldi P, Honda R, Nigg EA. Aurora-A overexpression reveals tetraploidization as a major route to centrososme amplification in p53-/- cells. EMBO J 2002;21:48392.

46. Rojanala S, Han H, Munoz RM, Browne W, Nagle R, Von Hoff DD, et al. The mitotic serine threonine kinase, Aurora-2, is a potential target for drug development in human pancreatic cancer. Mol Cancer Ther 2004;3:451-7.

47. Li D, Zhu J, Firozi PF, Abbruzzese JL, Evans $\mathrm{DB}$, Cleary $\mathrm{K}$, et al. Overexpression of oncogenic STK15/BTAK/Aurora A kinase in human pancreatic cancer. Clin Cancer Res 2003;9: 991-7.

48. Jeng YM, Peng SY, Lin CY, Hsu HC. Overexpression and amplification of Aurora-A in hepatocellular carcinoma. Clin. Cancer Res 2004;10:2065-71.

49. Hoque A, Carter J, Xia W, Hung MC, Sahin $\mathrm{AA}$, Sen $\mathrm{S}$, et al. Loss of Aurora A/STK15/BTAK overexpression correlates with transition of in situ to invasive ductal carcinoma of the breast. Cancer Epidemiol. Biomarkers Prev 2003;12:151822.

50. Moreno-Bueno G, Sanchez-Estevez C, Cassia R, Rodriguez-Perales S, DiazUriarte R, Dominguez 0, et al. Differential gene expression profile in endometrioid and nonendometrioid endometrial carcinoma: STK15 is frequently overexpressed and amplified in nonendometrioid carcinomas. Cancer Res 2003;63:5697-702.

51. Gritsko TM, Coppola D, Paciga JE, Yang L, Sun M, Shelley SA, et al. Activation and overexpression of centrosome kinase BTAK/Aurora-A in human ovarian cancer. Clin. Cancer Res 2003;9:1420-6.

52. Reichardt W, Jung V, Brunner C, Klein A, Wemmert S, Romeike BF, et al. The putative serine/threonine kinase gene STK15 on chromosome $20 \mathrm{q} 13.2$ is amplified in human gliomas. Oncol Rep 2003;10:12759.

53. Hamada M, Yakushijin Y, Ohtsuka M, Kaki-moto M, Yasukawa M, Fujita S. Aurora2/BTAK/STK15 is involved in cell cycle checkpoint and cell survival of aggressive non-Hodgkin's lymphoma. Br J Haematol 2003;121:439-47.

54. Dewar H, Tanaka K, Nasmythm K, Tanaka TU. Tension between two kinetochores suffices for their bi-orientation on the mitotic spindle. Nature 2004;428:93-7.

55. Murata-Hori M, Wang Y. The kinase activity of aurora $B$ is required for kinetochoremicrotubule interactions during mitosis. Current Biology 2002;12:894-99.

56. Araki K, Nozaki K, Ueba T, Tatsuka M, Hashimoto N. High expression of Aurora-
B/Aurora and Ipll-like midbody-associated protein (AIM-1) in astrocytomas. J Neuro oncol 2004;67:53-64.

57. Katayama H, Ota T, Jisaki F, Ueda Y, Tanaka T, Odashima S, et al. Mitotic kinase expression and colorectal cancer progression. J Natl Cancer Inst 1999;91: 1160-2.

58. Sorrentino R, Libertini S, Pallante PL, Troncone G, Palombini L, Bavetsias V, et al. Aurora B overexpression associates with the thyroid carcinoma undifferentiated phenotype and is required for thyroid carcinoma cell proliferation. J Clin Endocrinol Metab 2005; 90:928-35.

59. Sasai K, Katayama H, Stenoien DL, Fujii S, Honda R, Kimura M, et al. Aurora-C kinase is a novel chromosomal passenger protein that can complement Aurora B kinase function in mitotic cells. Cell Motility and the Cytoskeleton 2004;59:249-63.

60. Kimura M, Matsuda Y, Yoshioka T, Okano Y. Cell cycle-dependent expression and centrosome localization of a third human aurora/Ipl1-related protein kinase, AIK3. J Biol Chem 1999;274:7334-40.

61. Takahashi T, Futamura M, Yoshimi N, Sano J, Katada M, Takagi Y, et al. Centrosomal kinases, HsAIRK1 and HsAIRK3, are overexpressed in primary colorectal cancers. Jpn J Cancer Res 2000; 91:1007-14.

62. Giles FJ, Cortes J, Jones D, Bergstrom D, Kantarjian H, Freedman SJ. MK-0457, a novel kinase inhibitor, is active in patients with chronic myeloid leukemia or acute lymphocytic leukemia with the T315I BCRABL mutation. Blood 2007;109:500-2.

63. Modugno M, Casale E, Soncini C, Rosettani P, Colombo R et al. Crystal structure of the T315I Abl mutant in complex with the aurora kinases inhibitor PHA739358. Cancer Res 2007;67:7987-90.

64. Tokarski JS, Newitt JA, Chang CYJ, et al. The structure of dasatinib (BMS-354825) bound to activated ABL kinase domain elucidates its inhibitory activity against imatinibresistant ABL mutants. Cancer Res 2006;66:5790-7. 